\title{
Non-planar structure of analytical QCD predictions for the Gottfried sum rule
}

\author{
A.L.Kataev ${ }^{* \dagger}$ \\ Institute for Nuclear Research of the Russian Academy of Sciences, 117312 Moscow, Russia \\ E-mail: kataev@ms2.inr.ac.ru
}

\begin{abstract}
It is stressed that within large $N_{c}$-expansion analytical calculations of the $\left(\alpha_{s} / \pi\right)^{2} \mathrm{QCD}$ contributions to the valence part of the Gottfried sum rule for $F_{2}$ structure function of charged leptonsnucleon deep-inelastic scattering reveals the existence of the $O\left(1 / N_{c}^{2}\right)$ non-planar corrections only and the disappearance of the planar $O\left(N_{c}^{0}\right)$ perturbative terms. The relation between Gottfried and Adler sum rule for neutino-nucleon DIS is established and the proposal that the differebce between corresponding QCD corrections to higher non-singlet moments in charged-lepton and neutrino DIS are suppressed by $1 / N_{c}^{2}$ is made. The possible consequence of the cancellation of perturbative planar graphs in the considered perturbative series, namely the existence of light-quark flavour asymmetry $\bar{u}(x)<\bar{d}(x)$, is mentioned. The effect of the similar origin, i.e. the relation of "light-by-light-type"-type structure in DIS characteristics at the $\alpha_{s}^{3}$-level to the generation of light quark-antiquark asymmetry, is also commented.
\end{abstract}

XI International Workshop on Advanced Computing and Analysis Techniques in Physics Research April 232007

Amsterdam, the Netherlands

\footnotetext{
${ }^{*}$ Speaker.

${ }^{\dagger}$ Supported in part by RFBR Grants N 05-01-00992, N 06-02-16659
} 


\section{Introduction}

Modern calculation physics has several important features, which may push ahead this part of science. To our point of view among them are:

1. the requirement of reliability of the complicated numerical or analytical calculations, related to theelimination of bugs in the computer codes and final results. It seems to be obvious to realize this requirement, but sometimes not simple (see e.g. [1]);

2. the requirement to avoid shortcomings of definite numerical calculations, which may manifest themselves in the non-proper rounding off errors in the numerical results (see e.g.[1], [2]);

3. the interest to phenomenological applications of various perturbative results and the development of different resummations approaches of perturbative series (see e.g. [3], [4]);

4. the appearance of strong intention to go beyond straightforward theoretical calculations and the desire to find new mathematical [5] and physical [6] properties, related to the differences in the topological structure of various multiloop Feynman diagram, which define special analytical structures of perturbative series under investigation.

I will try to demonstrate that continuing at present QCD calculations of characteristics of deepinelastic scattering (DIS) satisfy all requirements mentioned above. In particular, I will concentrate myself on discussions of possible relations of the concrete analytical structures in the concrete results of perturbative QCD calculations to the existence in nature of definitely speaking non-perturbative effects, namely light quarks flavour asymmetry $\bar{u}(x)<\bar{d}(x)$ (see Ref.[6]) Related phenomenological manifestations of this effects will be also considered. Presented in Ref.[6] conjecture about existence of differences between QCD corrections to the non-singlet (NS) moments of charged leptons - nucleon and neutrino-nucleon DIS, which manifest themselves in the large $N_{c}$-limit, proposed in Ref.[8], will be described.The perturbative indications to the generation of $s(x)-\bar{s}(x)$ strange quark asymmetry (see Ref.[7] will be mentioned as well.

\section{Definitions of some perturbative series in DIS}

Let me follow normalization conditions of the related perturbative series, used in the work of Ref. [9] devoted to the detailed fits of the experimental data for the NS $x F_{3}$ SF of $v N$ DIS, provided by CCFR collaboration [10]. Consider DGLAP equation in the NS case

$$
Q^{2} \frac{d}{d Q^{2}} F^{N S}\left(x, Q^{2}\right)=\frac{1}{2} \int_{x}^{1} \frac{d z}{z}\left[P_{N S}\left(z, a_{S}\right)+\beta\left(a_{s}\right) \frac{\partial \ln C_{N S}\left(z, a_{s}\right)}{\partial a_{s}}\right] F^{N S}\left(\frac{x}{z}, Q^{2}\right)
$$

where $C_{N S}\left(z, a_{s}\right)$ and $P_{N S}\left(z, a_{s}\right)$ are the NS combinations of the coefficient functions and splitting functions, $F^{N S}$ - NS combinations of $F_{2}^{l, v}$ structure functions (SFs) of charged leptons $(l)$ or $v$ nucleon DIS. The QCD $\beta$-function is defined as

$$
\mu \frac{\partial a_{s}}{\partial \mu}=\beta\left(a_{s}\right)=-2 \sum_{n \geq 0} \beta_{n} a_{s}^{n+2}
$$


where $a_{s}=\alpha_{s} /(4 \pi)$ and the first two scheme-independent coefficients are normalized as

$$
\begin{aligned}
& \beta_{0}=\left(\frac{11}{3} C_{A}-\frac{2}{3} N_{F}\right) \\
& \beta_{1}=\left(\frac{34}{3} C_{A}^{2}-2 C_{F} N_{F}-\frac{10}{3} C_{A} N_{F}\right)
\end{aligned}
$$

The general expressions for the Casimir operators of the $S U\left(N_{c}\right)$ group of colour are $C_{A}=N_{c}$, $C_{F}=\left(N_{c}^{2}-1\right) /\left(2 N_{c}\right)$, while $N_{F}$ is the number of quarks flavours. The series for the coefficient functions and splitting functions of Eq.(2.1) read

$$
\begin{aligned}
C_{N S}\left(z, a_{s}\right) & =\sum_{n \geq 0} C_{n}^{N S}(z) a_{s}^{n} \\
P_{N S}\left(z, a_{S}\right) & =\sum_{n \geq 0} P_{N S}^{(n)}(z) a_{s}^{n+1}
\end{aligned}
$$

The corresponding non-expanded in $a_{s}$ NS splitting functions we will be interested in have the form, given in the work of Ref.[11]

$$
\begin{gathered}
P_{N S}^{ \pm}(x)=P_{q q}^{v}(x) \pm P_{q \bar{q}}^{v}(x) \\
P_{q_{i} q_{k}}=P_{\bar{q}_{i} \bar{q}_{k}}=\delta_{i k} P_{q q}^{v}+P_{q q}^{s} \\
P_{q_{i} \bar{q}_{k}}=P_{\bar{q}_{i} q_{k}}=\delta_{i k} P_{q \bar{q}}^{v}+P_{q \bar{q}}^{s} .
\end{gathered}
$$

where the next-to-next-to-leading order (NNLO) $a_{s}^{3}$ corrections to the NS combinations of valence $\left(P_{q q}^{v}, P_{q \bar{q}}^{v}\right)$ and sea $\left(P_{q q}^{s}(x), P_{q \bar{q}}^{s}\right)$ contributions were derived analytically in [11].

These results are related to the coefficients of the anomalous dimensions of NS moments through the following Mellin transformation

$$
\gamma_{N S}^{N}\left(a_{s}\right)=\sum_{n \geq 0} \gamma_{n}^{N} a_{s}^{n+1}=-2 \int_{0}^{1} d x\left[P_{N S}\left(x, a_{s}\right)\right] x^{N-1}=-2 \int_{0}^{1} d x\left[\sum_{n \geq 0} P_{N S}^{(n)}(x)\right] a_{s}^{n+1} x^{N-1}
$$

In the case of $\mathrm{N}=2,4,6,8$ Mellin NS moments of the charged lepton-nucleon DIS SF $F_{2}$ they were previously calculated analytically at the NNLO in Ref. [18], N=10 NNLO results were obtained in Ref.[19], while for $\mathrm{N}=12,14$ they are known from the calculations of Ref.[20]. In the case of $\mathrm{N}=16$ NS moment of $F_{2}$ SF the direct calculation of the $O\left(a_{s}^{3}\right)$ approximation for $\gamma_{N S}^{N}\left(a_{s}\right)$, performed in Ref. [21], helped to check the three-loop results for $P_{N S}\left(x, a_{s}\right)$ [11] by verifying coincidence of anomalous dimension of $\mathrm{N}=16$ moment, calculated in Ref.[21], with the result obtained from the expression of Ref.[11] using Mellin transformation in Eq.(2.10).

It should be stressed, that among the first phenomenological applications of the results of Refs.[18],[19] were the NNLO DIS fits of Refs.[12],[13], [14],[15],[16], [17], where the works of Refs,[12], [15] were devoted to the analysis of $F_{2}$ SF data. The works of Refs.[13],[14], [16],[17] were aimed to the "approximate" NNLO fits of CCFR $x F_{3}$ data for $v N$ DIS, which were essentially based on the results of rather complicated and, to my point of view, distinguished calculations of the NNLO corrections $C_{2}^{x F_{3}}(x)$ to the coefficient function of the DGLAP equation for $x F_{3}$ SF of $v N$ DIS, performed by van Neerven and Zijlstra [22]. The first confirmation of this result came from 
the coincidence of its first Mellin moment with the NLO analytical approximation for the GrossLlewellyn Smith sum rule, evaluated by Gorishny and Larin [23]. More detailed cross-checks were done in Ref.[24] using different methods.

The concrete theoretical arguments, that for moderate and high $\mathrm{N}$ the NNLO approximations of the NS anomalous dimensions for $F_{2}$ SF will be rather closed to the NNLO approximations of NS anomalous dimensions for $x F_{3}$ SF were used in Ref. [13]. Theoretical studies of these arguments, which in part are based on the proposal to use extrapolation procedure from even to odd values of $\mathrm{N}$ [12], are on the agenda (careful checks of the validity of the extrapolations procedures was done in Ref.[9] and in the resent work of Ref.[25]).

The "approximate" feature of the NNLO fits of $x F_{3}$ data pushed a-head analytical calculations of the NNLO approximations for NS anomalous dimensions and coefficient functions of $x F_{3}$ SF of odd Mellin moments with $\mathrm{N}=1,3,5,7,9,11,13$ [20] (the $N=1 \mathrm{NNLO}$ results of Ref.[20] coincide with the NNLO calculations for the Gross-Llewellyn Smith sum rule [26]).

Let us now return to the definition of the coefficient function in Eq.(2.5) in the case of NS combinations of $F_{2} \mathrm{SF}$. The NNLO term of order $a_{s}^{2}$ can be divided in two parts

$$
C_{2}^{N S, F_{2}}(x)=C^{(2),(+)}(x) \pm C^{(2),(-)}(x)
$$

where the sign plus in between two terms corresponds to the NS contribution to the coefficient function of the $F_{2} \mathrm{SF}$ of charged lepton-nucleon DIS, while the sign minus appears, when the coefficient function of the NS contributions to $F_{2}$ SF of neutrino-nucleon DIS is considered. The expressions for the terms $C^{(2),(+)}(x)$ and $C^{(2),(-)}(x)$, were calculated in Ref.[27] and confirmed in Ref.[24]. Thus, all discussions presented above, are demonstrating that the first requirement, mentioned in the Introduction, i.e. the reliability of the results obtained, is fulfilled in the case of the complicated NNLO calculations of DIS characteristics. The third requirement, and in particular, its first part, namely the applications of new perturbative results for the phenomenological studies, is also fulfilled in the case of DIS characteristics.

Indeed. the appearance of the calculations of Ref.[20] gave the possibility to perform more careful analysis of CCFR $x F_{3}$ structure functions data, which came from the studies of $v N$ DIS process at Tevatron. These phenomenological fits were made in Refs.[29], [9],[30],[31], [32]) using different theoretical approaches. Note, however, that after these works the number of questions still remain for possible future studies. For example, in spite of the fact, that the NNLO theoretical inputs of various fitting codes of analyzing DIS experimental data ( i.e. the ones, based on application of the DGLAP equation and on the reconstruction of structure functions from their moments using Bernstein and Jacobi polynomials) are coordinated, the outputs sometimes differ drastically.

Among the concrete questions is the existence of "small" NNLO value $\alpha_{s}\left(M_{Z}\right)=0,1142 \pm$ 0.0008 obtained by Bernstein polynomial analysis of the CCFR $x F_{3}$ measurements [31], which is agreement with "small" NNLO result $\alpha_{s}\left(M_{Z}\right)=0.1141 \pm 0,0014$ of the combined NNLO fits of various DIS data [33] (other "small" NNLO values were recently discussed in Ref.[34]), but is not consistent with the "world average" result $\alpha_{s}\left(M_{z}\right)=0.1189 \pm 0.0010$ [35]. It is also worth to mention, that, that the "small" result of Ref.[31] does not also agree with other NNLO applications of the Bernstein polynomial technique for studies of the same data CCFR $x F_{3}$ data, which give the following results $\alpha_{s}\left(M_{Z}\right)=0.1166 \pm 0.0013$ [29], $\alpha_{s}\left(M_{Z}\right)=0.1196_{-0.0031}^{+0.0027}$ [30] and $\alpha_{s}\left(M_{Z}\right)=0.1189 \pm 0.0019$ [32]. It will be of interest to trace the origin of these disagreements of 
applications of the same data and same theoretical method in the different works on the subject. Moreover, "small" NNLO $\alpha_{s}\left(M_{Z}\right)$ values, extracted from various characteristics of DIS, do not agree with the the recent NNLO result $\alpha_{s}\left(M_{Z}\right)=0.119 \pm 0.002$ (exp) \pm 0.003 (theory), obtained within the updated set of NNLO parton distributions of UK group [36]. It should be stressed, that this value is in perfect agreement with the one, obtained from the NNLO fits of $x F_{3}$ CCFR data using Jacobi polynomial technique, namely $\alpha_{s}\left(M_{Z}\right)=0.119 \pm 0.005$ (exp) \pm 0.003 (theory) [9], where experimental error is dominated by systematic uncertainties of CCFR $v N$ scattering DIS data. We think that more careful coordination of theoretical and experimental uncertainties of different NNLO determinations of $\alpha_{s}\left(M_{Z}\right)$ from the characteristics of DIS data will help to resolve existing disagreements.

\section{Adler and Gottfried sum rules: the definitions}

Two other sections are following the original presentation at ACAT07 Workshop of the materials of the work, made in collaboration with Broadhurst and Maxwell (see Ref.[6] ). Some additional considerations [37], which are supporting the results of Ref.[6] will be also commented.

The isospin Adler sum rule [38] is expressed through the structure function $F_{2}^{v N}$ of neutrinonucleon DIS as

$$
I_{A}=\int_{0}^{1} \frac{d x}{x}\left[F_{2}^{v p}\left(x, Q^{2}\right)-F_{2}^{v n}\left(x \cdot Q^{2}\right)\right]=4 I_{3}=2 .
$$

In terms of parton distributions Eq. (3.1) takes the following form

$$
I_{A}=2 \int_{0}^{1} d x\left[u_{v}(x)-d_{v}(x)\right]=2
$$

where $u_{v}(x)=u(x)-\bar{u}(x)$ and $d_{v}(x)=d(x)-\bar{d}(x)$ are the valence parton distributions. It was shown, that $I_{A}$ receives neither perturbative nor non-perturbative $\left(1 / Q^{2}\right)$-corrections [39]. In view of this the Adler sum rule is $Q^{2}$ independent and demonstrates the consequence of the property of scaling [40]. This property (or so called "automodelling" behavior of structure functions) was rigorously proved in the case of charged lepton-nucleon DIS [41] with application of general principles of local quantum field theory, described e.g. in the classical text-book [42]. It should be stressed, that the validity of this sum rule is supported by the existing neutrino-nucleon DIS data, which show no significant $Q^{2}$ variation in the range $2 \mathrm{GeV}^{2} \leq Q^{2} \leq 30 \mathrm{GeV}^{2}$ and give [43]

$$
I_{A}^{\exp }=2.02 \pm 0.40
$$

Though the error-bars are quite large, the precision could in principle may be improved by analyzing data of other $v N$ DIS experiments in more detail.

However, it is known, that in QCD scaling is violated. The sources of its violation are related to the asymptotic freedom effects and non-perturbative contributions. Both types of these effects manifest themselves (though is some puzzling way) in the analog of the Adler sum rule, namely in the Gottfried sum rule. It can be defined as the first $N=1$ NS Mellin moment of the difference of $F_{2}$ SFs of DIS of charged leptons on proton and neutron, namely

$$
I_{G}^{v}=\int_{0}^{1} \frac{d x}{x}\left[F_{2}^{l p}\left(x, Q^{2}\right)-F_{2}^{l n}\left(x \cdot Q^{2}\right)\right]=\frac{1}{3} \int_{0}^{1} d x\left(u_{v}\left(x, Q^{2}\right)-d_{v}\left(x, Q^{2}\right)\right)=\frac{1}{3}
$$


The definition of Eq. (3.4) is presented in the case of assumption accepted previously that the sea quarks are flavour-independent. It corresponds to the condition $\bar{u}\left(x, Q^{2}\right)=\bar{d}\left(x, Q^{2}\right)$, accepted in the early works on the subjects. However, due to the appearance of experimental data for the muonnucleon DIS, Drell-Yan process and semi-inclusive DIS we know at present that this condition is violated and $\bar{u}\left(x, Q^{2}\right)<\bar{d}\left(x, Q^{2}\right)$ ( for reviews see, e.g. [44]-[46]). Therefore, the definition of the Gottfried sum rule was modified as:

$$
I_{G}=\int_{0}^{1} \frac{d x}{x}\left[F_{2}^{l p}\left(x, Q^{2}\right)-F_{2}^{l n}\left(x \cdot Q^{2}\right)\right]=I_{G}^{v}+\frac{2}{3} \int_{0}^{1} d x\left(\bar{u}\left(x, Q^{2}\right)-\bar{d}\left(x, Q^{2}\right)\right),
$$

where the last term is related to the manifestation of isospin-breaking effects in the Dirac sea. Without it the valence part of the Gottfried sum rule of Eq. (3.4) contradicts the existing result of the analysis of muon-nucleon DIS data, performed by the NMC collaboration, namely [47]:

$$
I_{G}\left(Q^{2}=4 \mathrm{GeV}^{2}\right)=0.235 \pm 0.026 .
$$

Note, however, that the error of this experimental result may be over two times larger due to more careful treatment of the uncertainty from the small $x$ region [48]. In spite of this, definite contradictions of theory with the quark-parton result still exist [47] and it is worth to study the contributions of the perturbative and non-perturbative corrections to the valence part $I_{G}^{v}$.

\section{Large $N_{c}$-expansion of the Gottfried sum rule and its relation to the Adler sum rule.}

Let us now discuss the results of calculations of perturbative QCD corrections to the valence part of the Gottfried sum rule, following the considerations of Ref. [6] and using the ideology of large- $N_{c}$ limit, proposed in Ref.[8]. The solution of the renormalization group equation for the valence contribution $I_{G}^{v}$ to the Gottfried sum rule has the following form

$$
I_{G}^{v}=A\left(\alpha_{s}\right) C^{(l)}\left(\alpha_{s}\right)
$$

with the anomalous-dimension term

$$
\begin{aligned}
A\left(a_{s}\right) & =\exp \left[-\int_{\delta}^{a_{s}\left(Q^{2}\right)} \frac{\gamma_{N S}^{(l) N=1}(x)}{\beta(x)} d x\right]=1+\frac{1}{8} \frac{\gamma_{1}^{(N=1)}}{\beta_{0}}\left(\frac{\alpha_{s}}{\pi}\right) \\
& +\frac{1}{64}\left(\frac{1}{2} \frac{\left(\gamma_{1}^{(N=1)}\right)^{2}}{\beta_{0}}-\frac{\gamma_{1}^{(N=1)} \beta_{1}}{\beta_{0}^{2}}+\frac{\gamma_{2}^{(N=1)}}{\beta_{0}}\right)\left(\frac{\alpha_{s}}{\pi}\right)^{2}+O\left(\alpha_{s}^{3}\right)
\end{aligned}
$$

The anomalous dimension function of $N=1$ moment is defined as

$$
\gamma_{N S}^{(l) N=1}=\sum_{n \geq 0} \gamma_{i}^{(l) N=1} a_{S}^{i+1}
$$

The first coefficient $\gamma_{0}^{(l) N=1}$ is identically equal to zero [49]. The leading correction to Eq.(4.3) comes from the scheme-independent two-loop contribution [50]

$$
\gamma_{1}^{(l) N=1}=-4\left(C_{F}^{2}-C_{A} C_{F} / 2\right)[13+8 \zeta(3)-12 \zeta(2)]
$$


confirmed later on in Ref.[51], Notice the appearance of the distinctive non-planar colour factor $\left(C_{F}^{2}-C_{A} C_{F} / 2\right)=O\left(N_{c}^{0}\right)$, which exhibits $O\left(1 / N_{c}^{2}\right)$ suppression at large- $N_{c}$, in comparison with the individual weights of planar two-loop diagrams, namely $C_{F}^{2}$ and $C_{F} C_{A}$, that are canceling in the expression for $\gamma_{1}^{(l) N=1}$.

The three-loop coefficients of anomalous dimensions of N-th moments moments for the NS combinations of structure functions $F_{2}$ of neutrino - nucleon and charged lepton-nucleon DIS are related to the Mellin moments from the splitting functions as [11]

$$
\begin{aligned}
\gamma_{2}^{(v) N} & =2 \int_{0}^{1} d x\left[P_{N S}^{(2)-}(x)+P_{N S}^{(2)+}(x)\right] x^{N-1} \\
\gamma_{2}^{(l) N} & =2 \int_{0}^{1} d x\left[P_{N S}^{(2)-}(x)-P_{N S}^{(2)+}(x)\right] x^{N-1} .
\end{aligned}
$$

Taking into account the nullification of anomalous dimension function for the Adler sum rule, one can derive the useful identity

$$
\gamma_{2}^{(l) N=1}=-2 \int_{0}^{1} d x P_{N S}^{(2)+}(x)
$$

Integrating the results Ref.[11] and taking into account mathematical analytical procedures of Ref.[52], in Ref.[6] the analytical expression for $\gamma_{2}^{(l) N=1}$ was obtained:

$$
\begin{aligned}
\gamma_{2}^{(l) N=1}= & \left(C_{F}^{2}-C_{A} C_{F} / 2\right)\left\{C_{F}[290-248 \zeta(2)+656 \zeta(3)-1488 \zeta(4)+832 \zeta(5)+192 \zeta(2) \zeta(3)]\right. \\
& +C_{A}\left[\frac{1081}{9}+\frac{980}{3} \zeta(2)-\frac{12856}{9} \zeta(3)+\frac{4232}{3} \zeta(4)-448 \zeta(5)-192 \zeta(2) \zeta(3)\right] \\
& \left.+N_{F}\left[-\frac{304}{9}-\frac{176}{3} \zeta(2)+\frac{1792}{9} \zeta(3)-\frac{272}{3} \zeta(4)\right]\right\} \\
\approx & 161.713785-2.429260 N_{F}
\end{aligned}
$$

Notice the appearance in $\gamma_{2}^{(l) N=1}$ of three non-planar factors, namely $C_{F}^{2}\left(C_{F}-C_{A} / 2\right)$ and $C_{F} C_{A}\left(C_{F}-C_{A} / 2\right)$ and $C_{F}\left(C_{F}-C_{A} / 2\right) N_{F}$. These results are generalizing the observation of nonplanarity of $\gamma_{1}^{(l) N=1}$-term of anomalous dimension function to three-loops and may be considered as the first non-obvious argument in favor of the correctness of definite results of Ref. [11].

Let us turn to the coefficient function of the valence contribution to the Gottfried sum rule $I_{G}^{v}$. It has the following form

$$
C^{(l)}\left(a_{s}\right)=\frac{1}{3}\left[1+\sum_{n=1}^{\infty} C_{n}^{(l) N=1}\left(\frac{\alpha_{s}}{\pi}\right)^{n}\right]
$$

Like in the case of the Adler sum rule, the first coefficient $C_{1}^{(l) N=1}$ is equal to zero (see e.g. Ref.[51]). The the second coefficients to the N-th Mellin moments of the NS combinations of structure functions $F_{2}$ of charged lepton-nucleon and neutrino-nucleon DIS are related to the Mellin moments from Eq.(2.11) as

$$
\begin{aligned}
C_{2}^{(l) N} & =3 \int_{0}^{1} d x\left[C^{(2),(+)}(x)+C^{(2),(-)}(x)\right] x^{N-1} \\
C_{2}^{(v) N} & =\frac{1}{2} \int_{0}^{1} d x\left[C^{(2),(+)}(x)-C^{(2),(-)}(x)\right] x^{N-1}
\end{aligned}
$$


In the case of $\mathrm{N}=1$ the explicit numerical integration of Eq.(4.10) gave the following result [53]

$$
C_{2}^{(l) N=1}=3.695 C_{F}^{2}-1.847 C_{A} C_{F}
$$

where $C^{\left.(2),{ }^{ \pm}\right)}(x)$ in Eq.(4.10) were taken from Ref.[27] with the choice of $\overline{\mathrm{MS}}$ renormalization scale $\mu^{2}=Q^{2}$. Note, that non fulfillment of the second requirement, mentioned in the Introduction, namely the non-proper rounding off errors of numerical integrations of Ref.[53] did not allow to realize that Eq. (4.12) has the same non-planar structure as the expression for $\gamma_{1}^{(l) N=1}$. This fact was demonstrated in Ref. [6], where the following analytical result for $C_{2}^{(l) N=1}$ was obtained

$$
C_{2}^{(l) N=1}=\left(C_{F}^{2}-C_{A} C_{F} / 2\right)\left[-\frac{141}{32}+\frac{21}{4} \zeta(2)-\frac{45}{4} \zeta(3)+12 \zeta(4)\right] .
$$

with the help of the Mellin representation

$$
C_{2}^{(l) N=1}=2 \times 3 \int_{0}^{1} d x\left[C^{(2),(-)}(x)\right]
$$

which follows from Eqs.(4.5), (4.6) and the requirement of absence of QCD corrections to the Adler sum rule.

Next, taking the large $N_{c}$ limit for Eq.(4.1), which presumes that the corrections to the coefficients of QCD $\beta$-function of Eq.(2.3) and of Eq.(2.4) take the following form

$$
\begin{aligned}
& \beta_{0}=\frac{11}{3} N_{c}\left(1+O\left(\frac{N_{F}}{N_{c}}\right)\right) \\
& \beta_{1}=\frac{34}{3} N_{c}^{2}\left(1+O\left(\frac{N_{F}}{N_{c}}\right)\right)
\end{aligned}
$$

and $\alpha_{s} /\left(\pi N_{c}\right) \sim 1 /\left(N_{c}^{2} \ln \left(Q^{2} / \Lambda^{2}\right)\right)$, we find that Gottfried and Adler sum rules are rfelated as

$$
I_{G}^{v}=\frac{2}{3} I_{A}\left(1+O\left(\frac{1}{N_{c}^{2}}\right)\right)
$$

This relation is scheme-independent. Indeed, the transformation of the $\alpha_{s}$-corrections in Eq. (4.1) to another $M S$-like scheme, can be done with the help of the shift

$$
\frac{\alpha_{s}\left(Q^{2}\right)}{\pi}=\frac{\alpha_{s}^{\prime}\left(Q^{2}\right)}{\pi}+\beta_{0} \Delta\left(\frac{\alpha_{s}^{\prime}\left(Q^{2}\right)}{\pi}\right)^{2}
$$

where $\Delta$ is the concrete $N_{c}$-independent number, which is defined by the logarithm from the ratio of regularization scales $\mu_{\overline{M S}}^{2}$ and $\mu_{M S-l i k e}^{2}$. Thus, the general $M S$-like scheme expression for the coefficient $C_{2}^{(l) N=1}$ takes the following form

$$
C_{2 M S-l i k e}^{(l) N=1}=C_{2}^{(l) N=1} \overline{M S}+\gamma_{1}^{N=1} \Delta
$$

where both $C_{2}^{(l) N=1} \overline{M S}$ and $\gamma_{1}^{N=1}$ contain the same non-planar group weight $C_{F}\left(C_{F}-C_{A} / 2\right)$ [37]. 
Keeping in mind the intriguing relation between the valence part of the Gottfried sum rule and the Adler sum rule, one may make a prediction, that the difference between NS moments of $F_{2}$ in charged-lepton and neutrino scattering will continue to satisfy the property of non-planar suppression [6]. For example the ratio

$$
R_{2}^{N} \equiv \frac{C_{2}^{(l) N}-6 C_{2}^{(v) N}}{C_{2}^{(l) N}+6 C_{2}^{(v) N}}=\frac{\int_{0}^{1} \mathrm{~d} x C^{(2),(-)}(x) x^{N-1}}{\int_{0}^{1} \mathrm{~d} x C^{(2),(+)}(x) x^{N-1}}
$$

takes the value $R_{2}^{N=1}=1$ at $N=1$, but for $N=2$, one cane obtained from Ref.[24]

$$
R_{2}^{N=2}=-\frac{0.50593104}{5.4183241 N_{c}^{2}-4 N_{c} N_{F}-8.4480127}
$$

which is negative and small in magnitude at large $N_{c}$, and is continuing to decrease rapidly with increasing of $\mathrm{N}$ (see discussions in Ref.[6]). So, we expect that the ratio

$$
\frac{6 C_{n}^{(v) N}}{C_{n}^{(l) N}}=1+O\left(\frac{1}{N_{c}^{2}}\right)
$$

is valid not only for 2 loops, but in higher orders of perturbation theory as well [6].

It should be stressed, that the results and conjectures of Ref.[6], which were discussed in this talk, already received several confirmations. Indeed, in Ref.[54] the analytical expressions of Eq.(4.8) and Eq.(4.13) were reproduced with the help of calculations, which are based on different theoretical technique. One more argument in favor of careful study of the topological structure of analytical perturbative series for characteristics of DIS [6] came from the recent analytical calculations of the NS moments of $v N$ DIS both at the $a_{s}^{2}$ and $a_{s}^{3}$ level [55], and in the confirmation of the special role of non-planar-type colour structures in higher corrections of the quantities considered. Thus, two works, presented at this workshop, satisfy the fourth requirement of importance of analytical calculations mentioned, in the Introduction, namely the importance of careful study of topological structute of perturbative QCD series.

\section{Large $N_{c}$-limit: where are the effects of $O\left(N_{c}^{0}\right)$ corrections to the Gottfried sum?}

First of all it it worth to stress that non-planar perturbative QCD corrections produce the following numerical results [6]

$$
\begin{aligned}
I_{G}^{V} & =\frac{1}{3}\left[1+0.035521\left(\frac{\alpha_{s}}{\pi}\right)-0.58382\left(\frac{\alpha_{s}}{\pi}\right)^{2}\right] \text { for } N_{F}=3 \\
& =\frac{1}{3}\left[1+0.038363\left(\frac{\alpha_{s}}{\pi}\right)-0.56479\left(\frac{\alpha_{s}}{\pi}\right)^{2}\right] \text { for } N_{F}=4
\end{aligned}
$$

which are higher than the results of different analysis of NMC data [47], though recently updated [48]. This discrepancy can be resolved by introducing to $I^{v}$ the isospin-breaking effects in the Dirac sea $\bar{u}(x)<\bar{d}(x)$ as in Eq.(3.5). It is interesting to note that within the large $N_{c}$-limit of the modified soliton model of Ref. [56], proposed in Ref. [57], in the planar approximation one can get non-perturbative contribution of over $O\left(N_{c}^{0}\right)$ [58]

$$
\frac{1}{2}\left(3 I_{G}-1\right)=\int_{0}^{1} d x(\bar{u}(x)-\bar{d}(x))=O\left(N_{c}^{0}\right)
$$


which is absent in the perturbative series for $I_{G}^{v}$. Thus, the $O\left(N_{c}^{0}\right)$-effects, which do not manifest themselves in perturbative sector, may reflect the existence of flavour-asymmetry terms in the nucleon sea. In the letter case the values of $I_{G}$ between 0.219 and 0.178 were obtained for a range of constituents quark mass between 350 and $420 \mathrm{MeV}$, in fair agreement with the announced NMC result $I_{G}^{\text {exp }}=0.235 \pm 0.026$ at $Q^{2}=4 \mathrm{GeV}^{2}$ [47].

To summarize, we would like to emphasize that the bridge might exist between the $O\left(N_{c}^{0}\right)$ corrections, which are absent in the perturbative sector, but are manifesting themselves in the the non-perturbative regime.

\section{Conclusions}

In this talk we would like to put extra attention on the importance of analytical multiloop analytical perturbative QCD calculations of characteristics of DIS. Indeed, the results of definite calculations reveal the existence of special topological structure in some physical quantities, related to phenomenology. Among them is the perturbative series for the Gottfried sum rule, which up to or$\operatorname{der} a_{s}^{2}$ corrections is defined by non-planar $O\left(1 / N_{c}^{2}\right)$ suppressed corrections only. It is interesting to understand, whether this typical non-planarity will continue in higher loops. The results of the talk of Ref. [55] indicate that definite conjectures made in Ref. [6] on continuation of non-planar suppression in the characteristics of $v N$ DIS at the $a_{s}^{3}$ level remain true. The possible physical explanation of this special structure of perturbative QCD series is the existence in the Dirac sea of isospin symmetry breaking effect, which is generating light quark flavour asymmetry. Another interesting physical feature of the similar origin is the perturbative generation of strange-antistrange asymmetry $s(x)-\bar{s}(x)$ in the nucleon sea [7] by NNLO corrections $N_{F}\left(P_{q q}^{s}-P_{q \bar{q}}^{s}\right)=a_{s}^{3} P_{N S}^{(2), s}$, where $P_{N S}^{(2), s}$ term, calculated in Ref.[11] is proportional to $d^{a b c} d_{a b c}$ colour structure and result from "lightby-light" scattering type diagrams. Thus, one may expect, that the appearance of new colour structures in the perturbative series for DIS may be really connected to new physical conclusions, which should be studied more carefully.

\section{Note added}

After appearence of this contribution in arXiV, physical results of Ref.[55] were submitted for publication [59]. In particular, it was shown by analytical calculation of Ref. [59], that the observed and discussed in Ref.[6] and this presentation property of non-planarity of the $\alpha_{s}^{2}$ correction to the Gottfried sum rule coefficient function of Eq.(4.9) remain valid at the $\alpha_{s}^{3}$-level also.

\section{Acknowledgements}

I would like to acknowledge constant interest to my works by my late colleague W. van Neerven, his constructive questions and comments, which started in 1990 and unfortunately were interrupted after our e-mail discussion at the end of January, 2007. I am grateful to D.J. Broadhurst and C.J. Maxwell for very productive collaboration and to S.I. Alekhin, S. Catani, J. Blumlein, A.V. Kotikov, S.A. Kulagin, G. Parente and C. Weiss for useful discussions, related to this report. It is the pleasure to thank the organizers of XI International Workshop in Advanced Computing 
and Analysis Techniques in Physics Research ACAT 2007, and in particular J.A.M. Vermaseren, for hospitality in Amsterdam. I also acknowledge CERN Theory Unit for invitation and providing excellent working conditions during the work on this contribution.

\section{References}

[1] L. Hutton, To what extent can we rely on the results of scientific calculations, talk at ACAT07, Amsterdam, April 23, 2007

[2] M. Morhac, Error free algorithms to solve special and general discrete systems of linear equations, talk at ACAT07, Amsterdam, April 23, 2007.

[3] M. Beneke, Phys. Rept. 317 (1999) 1.

[4] D. V. Shirkov and I. L. Solovtsov, Theor. Math. Phys. 150 (2007) 132.

[5] D. J. Broadhurst, Eur.Phys. J. C8 (1999) 311.

[6] D. J. Broadhurst, A. L. Kataev and C. J. Maxwell, Phys. Lett. B590 (2004) 76.

[7] S. Catani, D. de Florian, G. Rodrigo and W. Vogelsang, Phys.Rev.Lett. 93 (2004) 152003.

[8] G. 't Hooft, Nucl. Phys B72 (1974) 461.

[9] A. L. Kataev, G. Parente and A. V. Sidorov, Phys.Part.Nucl. 34 (2003) 20 [Fiz. Elem. Chast. Atom. Yadra 34 (2003) 43] [hep-ph/0106221 ].

[10] W. G. Seligman et al., Phys. Rev. Lett. 79 (1997) 1213.

[11] S. Moch, J. A. M. Vermaseren and A. Vogt, Nucl.Phys B688 (2004) 101.

[12] G. Parente, A. V. Kotikov and V. G. Krivokhizhin, Phys.Lett. B333 (1994) 190.

[13] A. L. Kataev, A. V. Kotikov, G. Parente and A. V. Sidorov, Phys.Lett. B388 (1996) 179.

[14] A. L. Kataev, A. V. Kotikov, G. Parente and A. V. Sidorov, Phys.Lett. B417 (1998) 374.

[15] J. Santiago and F. J. Yndurain, Nucl.Phys. B563 (1999) 45.

[16] A. L. Kataev, G. Parente and A. V. Sidorov, Nucl.Phys. B573 (2000) 405.

[17] S. A. Kulagin and A. V. Sidorov, Eur.Phys. J. A9 (2000) 261

[18] S. A. Larin, T. van Ritbergen and J. A. M. Vermaseren, Nucl.Phys. B427 (1994) 41.

[19] S. A. Larin, P. Nogueira, T. van Ritbergen and J. A. M. Vermaseren, Nucl.Phys. B492 (1997) 338.

[20] A. Retey and J. A. M. Vermaseren, Nucl.Phys. B604 (2001) 281.

[21] J. Blumlein and J. A. M. Vermaseren, Phys.Lett. B606 (2005) 130.

[22] E. B. Zijlstra and W. L. van Neerven, Phys. Lett. B297 (1992) 377.

[23] S. G. Gorishny and S. A. Larin, Phys.Lett. B172 (1986) 109.

[24] S. Moch and J. A. M. Vermaseren, Nucl.Phys. B573 (2000) 853.

[25] S. Moch and M. Rogal, Nucl.Phys.B782 (2007) 51.

[26] S. A. Larin and J. A. M. Vermaseren, Phys.Lett. B259 (1991) 345.

[27] W. L. van Neerven and E. B. Zijlstra, Phys.Lett B272 (1991) 127. 
[28] E. B. Zijlstra and W. L. van Neerven, Nucl.Phys B383 (1992) 525.

[29] J. Santiago and F. J. Yndurain, Nucl. Phys. B611 (2001) 447.

[30] C. J. Maxwell and A. Mirjalili, Nucl. Phys. B645 (2002) 298.

[31] A. N. Khorramian and S. Atashbar Tehrani, JHEP 0703 (2007) 051

[32] P. M. Brooks and C. J. Maxwell, Nucl. Phys. B780 (2007) 76.

[33] S. Alekhin, JETP Lett. 82 (2005) 628 [Pisma Zh. Eksp. Teor. Fiz. 82 (2005) 710].

[34] J. Blumlein, talk at the 15th Int. Workshop on DIS and Related Subjects (DIS2007), Munich, Germany April 17, 2007 [arXiv:0706.2430 [hep-ph] ].

[35] S. Bethke, Prog.Part.Nucl..Phys. 58 (2007) 351.

[36] A. D. Martin, W. J. Stirling, R. S. Thorne and G. Watt, Phys.Lett.B652 (2007) 292

[37] A. L. Kataev, Phys.Part.Nucl. 36 (2005) S168 [hep-ph/0412369 ].

[38] S. L. Adler, Phys.Rev. 143 (1966) 1114.

[39] Y. L. Dokshitzer, G. Marchesini and B. R. Webber, Nucl. Phys. B469 (1996) 93.

[40] J. D. Bjorken, Phys.Rev. 179 (1969) 1547.

[41] N. N. Bogolyubov, V. S. Vladimirov and A. N. Tavkhelidze, Teor. Mat. Fiz. 12 (1972) 3; ibid. 12 (1972) 305.

[42] N. N. Bogolyubov and D. V. Shirkov, Introduction to the Theory of Quantum Fields Nauka, Moscow $(1973,1976,1986)$.

[43] D. Allasia et al., Z. Phys C28 (1985) 312.

[44] S. Kumano, Phys.Rept. 303 (1998) 183.

[45] G. T. Garvey and J. C. Peng, Prog. Part. Nucl. Phys. 47 (2001) 203.

[46] A. L. Kataev, Proc. 11 Lomonosov Conference on Elementary Particle Physics, Moscow, MSU, August 2003; "Moscow 2003, Particle physics in laboratory, space and universe", World Scientific, p.194 [hep-ph/0311091]. .

[47] M. Arneodo et al. [New Muon Collaboration], Phys. Rev. D 50 (1994) 1.

[48] R. Abbate and S. Forte, Phys.Rev. D72 (2005) 117503.

[49] D. J. Gross and F. Wilczek, Phys.Rev. D8 (1973) 3633.

[50] D. A. Ross and C. T. Sachrajda, Nucl.Phys.B149 (1979) 497.

[51] G. Curci, W. Furmanski and R. Petronzio, Nucl.Phys. B175 (1980) 27.

[52] J. M. Borwein, D. M. Bradley, D. J. Broadhurst and P. Lisonek, Trans. Am. Math. Soc 353 (2001) 907.

[53] A. L. Kataev and G. Parente, Phys.Lett. B566 (2003) 120.

[54] A. V. Kotikov and V. N. Velizhanin, [hep-ph/0501274 ].

[55] M. Rogal, talk at ACAT07, Amsterdam, April 26, 2007;

S. Moch, M. Rogal and A. Vogt; DESY 07-048, 2007 (to appear).

[56] E. Witten, Nucl.Phys.B223 (1983) 433.

[57] D. Diakonov, V. Petrov, P. Pobylitsa, M. V. Polyakov and C. Weiss, Nucl. Phys. B480 (1996) 341.

[58] P. V. Pobylitsa, M. V. Polyakov, K. Goeke, T. Watabe and C. Weiss, Phys.Rev. D59 (1999) 034024.

[59] S. Moch, M. Rogal and A. Vogt, [arXiv:0708.3731 [hep-ph] . 